\title{
ACOLHIMENTO COM AVALIAÇÃO E CLASSIFICAÇÃO DE RISCO COMO FERRAMENTA DE GESTÃO: PERCEPÇÃO DOS ENFERMEIROS
}

\section{USER EMBRACEMENT WITH EVALUATION AND RISK CLASSIFICATION AS MANAGEMENT TOOL: NURSES' PERCEPTION}

\author{
Juliano Carvalho de Farias ${ }^{1}$, Camila Zorzolli Nebel ${ }^{2}$, Cristiane Lima de Moraes ${ }^{3}$, \\ Letícia Pilotto Casagranda ${ }^{4 *}$, Celmira Lange ${ }^{5}$, Fernanda dos Santos ${ }^{4}$ \\ ${ }^{1}$ Escola Técnica Estilo, Pelotas, Rio Grande do Sul, Brasil. ${ }^{2}$ Pronto Atendimento da Unimed, \\ Pelotas, Rio Grande do Sul, Brasil. ${ }^{3}$ Universidade Católica de Pelotas, Pelotas, Rio Grande \\ do Sul, Brasil. ${ }^{4}$ Programa de Pós Graduação da Faculdade de Enfermagem, Ciências da \\ Saúde, Universidade Federal de Pelotas. Pelotas, Rio Grande do Sul, Brasil. ${ }^{5}$ Faculdade de \\ Enfermagem. Universidade Federal de Pelotas. Pelotas, Rio Grande do Sul, Brasil
}

*Autor correspondente: Rua Gomes Carneiro 01, Sala: 202. E-mail: cissapc@yahoo.com.br. Telefone: (53) 3221-1523

\begin{abstract}
RESUMO
Objetivou-se conhecer a percepção do enfermeiro frente à utilização do Acolhimento com Avaliação e Classificação de Risco como uma ferramenta na gestão e acolhimento, no Pronto-Socorro de Pelotas/RS. Foi realizada uma Pesquisa qualitativa, exploratória e descritiva. As informações foram coletadas no período de setembro a outubro de 2010 por meio de entrevistas semiestruturadas. Os dados foram analisados por meio de análise temática. Os enfermeiros são possuidores de conhecimentos acerca desta ferramenta, e identificaram, ainda, como pontos positivos, a reorganização do sistema de saúde seguida de valorização do profissional e usuário; como desvantagens, a falta de comprometimento médico e a sobrecarga de trabalho da enfermagem. Concluiuse que reconhecer o acolhimento como elemento essencial no processo de trabalho da enfermagem é fundamental, pois a partir deste fazer é possível promover um atendimento humanizado e resolutivo, estabelecido pelo contato direto com o usuário. Descritores: Enfermagem; Acolhimento; Serviço hospitalar de emergência.
\end{abstract}

\begin{abstract}
It was aimed to know the nurse's perception towards the use of User Embracement with evaluation and risk classification as a management and welcoming tool in the first aid station of Pelotas/RS. It was used a qualitative methodology, exploratory and descriptive research. Information was collected from September to October 2010 through semi-structured interviews. Data was analyzed through thematic analysis. Nurses are possessors of knowledge about this tool, having identified as positive aspects the reorganization of the health system, followed by professional and user's enhancement; as disadvantage, the lack of medical commitment and the nurses' work overload. Acknowledge the welcoming as essential element in the process of work in nursing is fundamental. From this perspective, it is possible to promote a humanized and resolutive care, established by direct contact to the user.
\end{abstract}

Descriptors: Nursing; User Embracement; Emergency Service Hospital. 


\section{INTRODUÇ̃̃O}

A demanda expressiva por atendimentos de urgência e emergência tem crescido nos últimos anos, principalmente devido ao grande número de acidentes e violência urbana, o que constitui, desta forma, um importante componente de assistência à saúde no Brasil. Entretanto, a realidade vivenciada por muitos é a superlotação destes serviços, e um dos motivos para que aconteça este fato são os problemas organizacionais, como o atendimento por ordem de chegada, sem estabelecimento de critérios clínicos, agravando assim o atendimento a quem realmente necessita (SOUZA et al., 2011).

Nesse sentido, o Ministério da Saúde (MS), através da Portaria n. ${ }^{\circ}$ 2048/GM de 2002, regulariza o serviço de urgência e emergência e, em 2003, lança a Política Nacional de Humanização (PNH), que propõe a implantação do Acolhimento com Avaliação e Classificação de Risco como uma ferramenta de gestão do atendimento prestado neste serviço. Esse instrumento surge com a proposta de reorganização, promovendo ampla divulgação das informações referentes ao seu funcionamento para os usuários, assim como a realização de um acolhimento qualificado, que pressupõe uma resposta mais rápida, adequada e centrada na necessidade de cada indivíduo (BRASIL, 2002; JUNIOR, MATSUDA, 2012).

O Acolhimento com Avaliação e Classificação de Risco é criado com o objetivo de atender o paciente no momento da sua chegada, reduzindo assim o período de espera no atendimento. Essa ferramenta leva em consideração a necessidade e o risco de agravo à saúde do paciente. Ainda, promove o encaminhamento ao serviço de atenção primária ou especialidade, se estes forem a referência no momento da avaliação. Acredita-se que a implementação dessa ferramenta promoveria o descongestionamento nas portas de entrada dos serviços de PS, além de garantir um atendimento humanizado e acolhedor ao usuário, bem como aos seus familiares e/ou acompanhantes (JUNIOR, MATSUDA, 2012; BRASIL, 2004).

Nas emergências, a classificação de risco é uma nova área de atuação do enfermeiro. Além da experiência profissional, a padronização no atendimento permite ampliar a qualidade do serviço de enfermagem, aperfeiçoando a assistência e o gerenciamento da unidade.
Assim sendo, o enfermeiro é o profissional recomendado para realizar o Acolhimento com Avaliação e Classificação de Risco, por ser possuidor de um conhecimento clínico, habilidades específicas e atitudes necessárias para a implementação dessa ferramenta nos serviços de emergência, tendo garantido o exercício profissional que dispõe sobre a consulta de enfermagem como uma atividade privativa do enfermeiro (JUNIOR, MATSUDA, 2012).

Dessa forma, percebe-se que o enfermeiro estabelece uma dependência recíproca frente ao cuidado prestado durante toda a permanência do usuário nesse serviço, promovendo uma mudança na relação profissional/usuário, que se dá através de um acolhimento qualificado e centrado na identificação de necessidades de saúde, se utilizando de condutas técnicas, éticas e humanitárias. Com o conhecimento da percepção deste profissional, espera-se saber qual é o seu entendimento acerca do que a utilização dessa ferramenta traz de importante para o serviço, o usuário e o profissional, e as modificações nas relações humanas que ela ocasionará.

Portanto, o objetivo deste estudo é conhecer a percepção do enfermeiro frente à utilização do Acolhimento com Avaliação e Classificação de Risco como uma ferramenta na gestão e acolhimento, no Pronto-Socorro de Pelotas/RS.

\section{MATERIAL E MÉTODO}

Trata-se de uma pesquisa exploratória, descritiva e com uma abordagem qualitativa, a qual é voltada para conhecer a percepção de enfermeiros frente ao Acolhimento com Avaliação e Classificação de Risco. A pesquisa foi realizada com enfermeiros atuantes no serviço de Pronto-Socorro na cidade do estudo, localizada no sul do Rio Grande do Sul. A quantidade de participantes foi definida de acordo com o número de enfermeiros que trabalhavam neste serviço de pronto-socorro. Excluíram-se três enfermeiros por motivo de falha na gravação da entrevista. A amostra fina se compôs de oito enfermeiros, identificados com letras. Primeiramente usou-se a letra minúscula "e" referente a "enfermeiro", em seguida uma letra maiúscula, à escolha do entrevistado.

A coleta de dados aconteceu nos meses de setembro a outubro de 2010, por meio de entrevistas semiestruturadas e individuais, realizadas pelos pesquisadores, com duração média de 60 minutos. O local escolhido para as entrevistas foi uma sala dentro do 
serviço, a qual proporcionava um ambiente climatizado, tranquilo e favorável para os questionamentos necessários, sendo norteadas por questões abertas abordando os objetivos deste estudo. Bem como eram agendadas para um horário que ficasse adequado para cada participante.

A análise dos dados ocorreu por meio da análise temática, sendo a mais indicada para trabalhos qualitativos na área da saúde, visto que tem a finalidade de desvendar a essência do conteúdo que integra uma conversação, cuja repetição da ocorrência dos dados expresse algum episódio para o objeto do estudo. Este tipo de análise divide-se em três fases, são elas: pré-análise - consiste na seleção dos dados, revisão das hipóteses/pressupostos e dos objetivos iniciais da pesquisa, a partir da realização de leituras fluentes e exaustivas que permitam a saturação do conteúdo, bem como novos direcionamentos interpretativos; exploração do material - consiste basicamente na unificação dos assuntos, que se caracterizam por expressões ou palavras encontradas na voz dos sujeitos; e o tratamento dos resultados obtidos e interpretações - esta etapa caracteriza-se por realizar operações estatísticas ou complexas que colocam em destaque as informações obtidas, pois deste ponto de partida o pesquisador sugere uma dedução e realiza interpretações, inter-relacionando com o quadro teórico planejado primeiramente, possibilitando até novos rumos acerca de dimensões teóricas e interpretativas indicadas pela leitura do material (MINAYO, 2008).

Aos enfermeiros que aceitaram participar da pesquisa era entregue um Termo de Consentimento Livre e Esclarecido, em duas vias; a primeira via ficou com o entrevistado e a segunda, com os pesquisadores. Assegurou-se aos enfermeiros participantes a liberdade de desistirem da pesquisa a qualquer momento. A pesquisa seguiu os princípios da Resolução do Conselho Nacional de Saúde no 196/96 e teve aprovação do Comitê de Ética em Pesquisa da Universidade Católica de Pelotas, sob o Protocolo n. ${ }^{\circ}$ 2010/22 (BRASIL, 1996).

\section{RESULTADOS E DISCUSSÃO}

Os temas que surgiram foram: conhecimento dos enfermeiros acerca de Acolhimento num Serviço de Emergência e vantagens/desvantagens percebidas pelos enfermeiros no Acolhimento com Avaliação e Classificação de Risco. O primeiro tema faz referência às questões relacionadas ao conhecimento do enfermeiro a respeito do Acolhimento com Avaliação e Classificação de Risco num Serviço de Emergência. A partir deste assunto, percebeu-se que o enfermeiro entendia que esta ferramenta é uma forma de receber o paciente, identificar o seu problema de saúde, classificar de acordo com o protocolo e tentar encaminhá-lo para um atendimento organizado, promovendo desta forma um atendimento humanizado.

Os entrevistados salientaram a recepção como um dos elementos presentes nessa ferramenta. De acordo com as falas: “[...] entendo que é onde recebemos os pacientes que procuram atendimento no pronto-socorro [...](eZ)"; " [...] acolhimento é a recepção do paciente numa unidade de atendimento de pronto atendimento [...] (eR)".

Dessa maneira, os enfermeiros percebiam que o acolhimento não é entendido apenas como a solução do problema que chega ao serviço de urgência e emergência, mas também como assistência diferenciada e integral, com a qual, por meio da escuta qualificada e centrada da queixa principal do usuário, podem-se identificar as necessidades de saúde. Neste contexto, o enfermeiro, ao realizar o acolhimento, acaba sendo reconhecido dentro do serviço de saúde, tendo exultação ao realizar a sua tarefa, bem como ajudar na resolução do problema do usuário (WEYKAMP et al., 2015).

Nessa perspectiva, outros entrevistados referiram o acolhimento como um encaminhamento, assim descrito: "[...] na medida do possível, solucionar, encaminhar, não apenas fazer um bom recebimento, mas tentar na medida do possível solucionar e encaminhar as questões [...] (eP)"; “[...] identifica qual o seu problema para encaminhá-lo da melhor maneira [...] (eA)".

Quando se pensa em acolhimento, no contexto do SUS, sabemos que a orientação do usuário em relação ao atendimento de suas necessidades de saúde deveria ser atrelada às redes de atenção dos serviços nos três níveis de complexidade, em todas as áreas de atuação, num determinado território. Esse modelo de atenção propõe a criação de vínculos entre diferentes serviços, que vão desde a atenção básica até a alta complexidade, com o intuito de promover encaminhamentos que garantam a promoção, prevenção, recuperação e reabilitação das condições de vida e saúde de indivíduos e coletividades. A articulação entre os serviços de Urgência e Emergência (UE) e a rede de 
saúde é o que garante o acesso universal, integral e igualitário para os usuários do Sistema Único de Saúde (SUS), mediante a pactuação de ações para referência e contrarreferência (JUNIOR, MATSUDA, 2012).

Cabe reforçar que o acolhimento poderia servir como uma nova metodologia dos processos de trabalho em saúde. É a partir do uso dessa tecnologia que o profissional da saúde tem a possibilidade de escutar, coletar informações, avaliar e decodificar as necessidades de saúde do paciente, nos diferentes níveis de atenção, para que possa adotar uma postura coerente e eficaz na resolução dos problemas de saúde deste indivíduo, sua família e comunidade. É nesse sentido que se conhece a importância de se oferecer orientação aos usuários do sistema de saúde, para que tenham continuidade da assistência.

Alguns entrevistados relataram que a classificação é uma forma de identificar a demanda de saúde imediata do usuário do serviço, conforme os registros: “[...] é a classificação do cliente pela sua gravidade e da patologia, encaminhado da melhor forma para ser melhor atendido e mais rápido [...] (eA)"; " “...] basicamente é classificar por cores o nível de urgência do atendimento [...] (eR)"; "[...] eu acredito que a classificação é uma forma [...] de tu atender, de tu avaliar o paciente e saber se a vida dele está em risco naquele momento ou não [...] (eB)".

Observa-se na leitura da PNH que o Acolhimento com Avaliação e Classificação de Risco é uma ferramenta do cuidado, que tem como finalidade a execução de atividades que promovam uma ação eficaz na identificação das necessidades de saúde, priorizando atendimentos de acordo com o risco de agravo às condições de saúde. Nessa metodologia, os serviços de urgência e emergência priorizam o atendimento dos mais graves em relação aos menos graves, promovendo o encaminhamento das situações menos graves aos serviços pertinentes (BRASIL, 2004).

Destaca-se ainda que a implementação dessa ferramenta pode ser um elemento fundamental a fim de reorganizar os serviços e práticas de saúde, tornando-se peça-chave na mudança do cenário desta área. Destarte, a utilização da Avaliação com Classificação de Risco nos serviços de urgência e emergência permitiria a disseminação de informações claras com relação ao funcionamento destes, além de otimizar o atendimento pela estratificação dos casos mais graves, os quais devem ser priorizados, tornando o atendimento organizado e humanizado (BELUCCI JUNIOR, et al., 2015).

Nesse contexto, a organização e a humanização surgem na fala deste entrevistado, como referência aos benefícios originados por essa ferramenta do cuidado em UE, assim descrita: “[...] classificar risco é organizar e humanizar a forma de atendimento [...] quem está mais necessitado será atendido primeiro [...] (eP)".

Admitir a organização e a humanização como elementos presentes no Acolhimento com Avaliação e Classificação de Risco é imprescindível para o sucesso dessa ferramenta na reorganização dos serviços de saúde. Para tanto, são necessárias reflexão e problematização dos processos de trabalho, de modo que a equipe multiprofissional tenha essa compreensão. Essa reorganização engloba todos os níveis de complexidade do SUS, tendo em vista o delineamento das ações de referência e contrarreferência entre os serviços. Para isso, é fundamental que se promova ampla divulgação das informações referentes ao funcionamento e fluxo desta nova metodologia (BRASIL, 2002; JUNIOR, MATSUDA, 2012).

São várias as funções presentes na implementação e organização da Avaliação com Classificação de Risco, sendo prioritário o atendimento do paciente no momento em que chega ao serviço, para que seja realizada uma avaliação das condições de saúde e identificação das necessidades de acesso, de modo que este seja organizado para o atendimento de acordo com a gravidade e/ou risco de vida. Este fluxo interno de acolhimento é um fator importante na redução das filas e do tempo de espera nos serviços de UE (JUNIOR, MATSUDA, 2012; BRASIL, 2004).

Com relação à humanização, entende-se que seja reflexo do acolhimento, uma vez que acontece no momento em que o serviço garante acesso a todos os usuários que necessitam de algum tipo de atendimento. Mesmo que, num sentido mais amplo, algumas definições da palavra se confundam com a noção de um ambiente adequado, uma boa recepção administrativa e leitos confortáveis, sabe-se que esses elementos são necessários para garantir uma certa qualidade no serviço. Contudo, a humanização se relaciona diretamente ao modo como o usuário é ouvido, assistido e cuidado, por meio de um atendimento qualificado e capacitado, que garanta a resolução do seu problema de saúde (ARAUJO, et al., 2014). 
A humanização do atendimento deve ser compreendida pelos trabalhadores como parte do processo, porém não é componente desta tecnologia, mas sim resultado de um conjunto de ações resolutivas que acolhem o usuário e sua família, originando informações sobre todo o seu funcionamento, fluxos e serviços de referência que estão ligados à UE.

$\mathrm{O}$ segundo tema faz referência às vantagens e desvantagens percebidas pelos enfermeiros no Acolhimento com Avaliação e Classificação de Risco.

Em relação às vantagens percebidas pelos enfermeiros no Acolhimento com Avaliação e Classificação de Risco, eles indicaram a prioridade no atendimento, reorganização do sistema e a conscientização da população, assim expressadas como fatores positivos, conforme os registros: "[...] vantagem é toda para o paciente, em que os mais graves são atendidos mais rápidos sem ordem de chegada [...] (eA)"; "[...] a gente consegue priorizar os atendimentos de emergência, coisas que não tinham antes, então é vantajoso [...] (eR)"; “[...] vantagens como reorganizar a fila, tentar conscientizar a população de que aqui é um pronto-socorro e não um local para as pessoas virem fazer consultas $[\ldots](\mathrm{eR}) "$.

Nos serviços de emergência sempre prevaleceu a triagem de pacientes, mesmo que num processo de exclusão. A triagem tem como finalidade a seleção e escolha, e em nenhum momento o acolhimento e a classificação dos clientes. A metodologia proposta pelo MS, que permite acolher, avaliar e classificar os usuários, de acordo com o risco de agravamento das suas condições de vida e saúde, priorizando de acordo com a EU (BRASIL, 2009).

A classificação de risco define-se como um processo dinâmico para identificação das necessidades de saúde dos pacientes que chegam ao serviço de emergência, dando destaque para atendimento prioritário e imediato às situações que apresentem potencial de risco à vida, agravos à saúde e o sofrimento do usuário (BRASIL, 2004).

A reorganização das redes de saúde é uma das finalidades dessa ferramenta, o que recai diretamente nos serviços de saúde que as compõem, pois a implementação do Acolhimento com Avaliação e Classificação de Risco, nos serviços de UE, encontra-se alicerçada na rede que garantirá os encaminhamentos necessários para a diminuição no tempo de espera para atendimento, bem como a segurança de que a população terá resolutividade nos outros níveis de complexidade.

Para tanto, é necessário definir, por via municipal, como será formada a rede de atenção à saúde, nos três níveis de complexidade, para a definição dos fluxos de entrada e saída de usuários do SUS, a fim de garantir ações de referência e contrarreferência pactuadas pela gestão compartilhada entre o município e serviços de saúde (MOURA, et al., 2015).

Sabe-se, portanto, que muitos são os problemas que trazem o usuário ao serviço de UE. Dentre eles destacam-se a ausência de definições políticas, a baixa resolutividade dos serviços da atenção primária, a dificuldade de acesso às especialidades, entre outros que geram insegurança e descrença na população. Esses fatores, associados ou não, fazem com que os serviços de UE se tornem superlotados, e, acima de tudo, acabem se configurando em leitos de hospitais, para garantir o atendimento da grande maioria de casos que chegam, diariamente, em busca de resolutividade (FEIJÓ, et al., 2015).

Desse modo, oferecer as garantias que a implementação desta ferramenta traz a todos os níveis de complexidade, interessados na modificação do sistema de saúde, seria um fator determinante na conscientização dos usuários quanto à importância de buscar atendimento nos serviços de referência, para cada complexidade, sem que para isso seja necessário ir a um serviço de UE, na tentativa de reverter esta procura incessante pela emergência. Conscientizar e valorizar a população é fundamental durante a educação em saúde, enfatizar a importância da busca pela atenção primária, a fim de se criar vínculos entre usuário e unidade básica de saúde.

A valorização do paciente e da equipe de enfermagem foram pontos relevantes observados pelos sujeitos, assim destacados: “[...] vantagem maior é do cliente que chega até o serviço [...] que ele vai que [...] se for uma urgência, uma emergência realmente, naquele momento com certeza ele vai ser valorizado pela situação dele e também valoriza o serviço [...] (eB)"; “[...] a classificação de risco [...] como uma forma de atendimento [...] uma forma de gestão, ela vem valorizar isso que a enfermagem já fazia antes [...] (eB)".

Partindo do momento em que se aceita confrontar os problemas em defesa da vida, garantindo o direito à saúde, sabe-se que os desafios são muitos quanto ao compromisso que cada um de nós tem 
no reconhecimento do próximo como um ser multidimensional, que este deverá ser acolhido integralmente, ou seja, a partir de suas dores, alegrias, culturas, compreensão de seus sentimentos e o modo como se encontra na vida.

Assim, no campo da saúde, é fundamental que os profissionais busquem, continuamente, estratégias para promover um cuidado transcultural, integral e humanizado. A utilização de tecnologias, tais como o Acolhimento com Avaliação e Classificação de Risco, no cotidiano dos serviços de saúde, contribui para uma qualidade na assistência ao indivíduo, família e comunidade, garantindo condições de vida e saúde (BRASIL, 2009).

Com relação à valorização e à motivação profissional, percebe-se que são intrínsecas e, se existe a vontade de se alargar a operacionalidade através desta ferramenta de gestão e cuidados em saúde, os gestores devem estar atentos às necessidades dos trabalhadores, valorizando o ser/fazer. A transformação dos elementos que motivam os trabalhadores é influenciada pelo modo como a instituição os valoriza.

Quanto às desvantagens percebidas pelos enfermeiros no Acolhimento com Avaliação e Classificação de Risco, foi possível saber que a sobrecarga de trabalho ainda é vista como um fator negativo na implementação de novas práticas em saúde, como salienta o entrevistado: "[...] a enfermagem está muito sobrecarregada, acho que não é só eu que vejo isso, nós estamos com uma responsabilidade muito grande sobre os nossos ombros [...] e o nosso papel é importantíssimo [...] (eP)".

O enfermeiro em unidades de emergência desenvolve um grande volume de trabalho, tendo em vista a grande demanda e acúmulo de tarefas. Fazem parte do seu processo de trabalho o cuidado, a educação em saúde e a gestão do serviço de enfermagem, dentre os quais se salienta a assistência ao paciente em situação crítica, educação do paciente e família para a alta hospitalar, capacitação da equipe de enfermagem, planejamento, organização, implementação e avaliação das condições de trabalho. Perceber este processo na sua íntegra pressupõe a sobrecarga de trabalho destes profissionais (WEYKAMP, et al., 2015; AMESTOY, et al., 2016).

$\mathrm{Na}$ atualidade, o enfermeiro deve assumir o compromisso pela busca de uma vasta gama de conhecimentos que solidificam o cuidado na urgência e emergência, pois seu processo de trabalho se estende desde a realização procedimentos até o planejamento e execução de atividades mais complexas e especializadas (AMESTOY, et al., 2016).

$\mathrm{O}$ atendimento e comprometimento médico também foram observados como desvantagens neste processo, conforme mostram as falas: "[...] mas não temos o apoio médico, ainda não tem médico no Pronto Atendimento, então fica difícil, nós ficarmos (re)classificando os pacientes [...] (eR)"; “[ ...] amarelo, que deveria ser atendido em 20 e 30 minutos, ficam horas esperando; então, enquanto os médicos não se comprometerem a participar, não vai funcionar bem [...] (eR)".

Do mesmo modo que os sujeitos identificaram essa problemática, as cartilhas da PNH relatam a necessidade de descentralização das ações em saúde, as quais se referem ao papel que cada profissional da equipe deve e pode desempenhar, por ter competência e habilidade. Salientaram, ainda, que essa transformação deverá acontecer especificamente no fazer da medicina, que há muitos anos exerce papel central e hegemônico nos modelos de saúde. Atualmente, sabemos da importância do trabalho em equipes interdisciplinares, assim como as inter-relações que se estabelecem no fazer de cada profissional. Torna-se cada vez mais importante uma caminhada profissional que prime pela participação de todas as equipes na implementação do Acolhimento com Avaliação e Classificação de Risco nos serviços de UE, com o intuito de motivar e valorizar cada membro da equipe de saúde (BRASIL, 2004).

Outro ponto relevante, e não obstante ao anterior, se refere à comunicação, elemento imprescindível no cotidiano dos serviços de saúde, o qual foi destacado como empecilho no processo de implementação da classificação de risco, de acordo com o sujeito: "[...] uma desvantagem é a diferença de linguagem entre os profissionais [...] entre o médico e o enfermeiro, entre o técnico e enfermeiro [...] então, que não tenha a mesma linguagem $[\ldots](\mathrm{eB})$ ".

A comunicação é um elemento singular e ao mesmo tempo complexo, que exige atenção, observação e escuta eficaz, para que possa ser processada de maneira clara e objetiva entre as partes envolvidas nesse processo. Para tanto, na utilização do Acolhimento com Avaliação e Classificação de Risco, como uma tecnologia do cuidado presente nos serviços de UE, 
devem existir a qualificação e capacitação das equipes atuantes (BRASIL, 2004).

Dessa forma, esse processo de aprendizagem visa à padronização do atendimento, fato que exige o uso de uma linguagem única e acessível entre todos os envolvidos.

\section{CONSIDERAÇÕES FINAIS}

Percebe-se que os entrevistados compreendiam o Acolhimento com Avaliação e Classificação de Risco como um elemento fundamental no processo de trabalho da enfermagem. É a partir deste fazer que lhes é possível promover um atendimento mais humanizado e resolutivo, estabelecido pelo contato direto com o usuário. Na utilização dessa tecnologia, é possível acolher, avaliar, identificar necessidades de saúde, classificar de acordo com o risco de agravos à vida e saúde, bem como proceder ao atendimento e encaminhamento de cada situação, individualmente. Nesse sentido, os entrevistados evidenciaram que esta metodologia, presente no cotidiano dos serviços de UE, torna-se uma importante ferramenta de gestão e cuidado para a enfermagem/saúde.

Os enfermeiros relataram que a implementação da classificação de risco promoveu agilidade no tempo de espera dos usuários que chegaram à unidade, bem como o estabelecimento de metas que priorizaram o atendimento dos casos mais graves, tornando-o mais eficaz e resolutivo.

Com relação às vantagens, teve-se a prioridade no atendimento aos pacientes mais graves, a reorganização das filas de espera e do serviço de saúde, a conscientização da população sobre os fluxos de atendimento em nível de rede de atenção à saúde do município, bem como a valorização das necessidades de saúde dos pacientes, e a valorização dos profissionais de enfermagem. Destacaram-se como desvantagens a sobrecarga de trabalho desta equipe de profissionais, a falta de comprometimento da equipe médica e a comunicação estabelecida entre as diferentes equipes de trabalho, que interferem na padronização do fluxo de atendimento.

Por fim, consideramos que este estudo tenha sido de extrema relevância para os profissionais que atuam neste serviço de UE do Município de Pelotas/ RS, pois servirá de subsídio para uma avaliação e (re)organização das práticas e processos de trabalho da equipe de enfermagem e saúde. Para a academia, servirá de base para levantar lacunas que possibilitem a elaboração e realização de novas pesquisas acerca desta temática, buscando contribuir cada vez mais com a qualidade da assistência prestada aos usuários do SUS, e, consequentemente, favorecendo ações de promoção, prevenção e recuperação das condições de vida e saúde da população.

\section{REFERÊNCIAS}

AMESTOY, S. C.; LOPES, R. F.; SANTOS, B. P.; DORNELLES, C.; FUCULO JUNIOR, P. R. B.; SANTOS, E. A. Exercício da liderança do enfermeiro em um serviço de urgência e emergência. Revista Eletrônica Gestão e Saúde. v.7, n.1, p.38-51, 2016.

ARAUJO, Y. B.; FERREIRA, L. B. A.; SANTOS, C. M.; SILVA, A. T. M. F.; GOMES, M. S. M. Acolhimento e classificação de risco nos serviços de urgência e emergência: limites e possibilidades uma questão para os enfermeiros. Perspectivas Online: Ciência Biológicas e da Saúde. v.15, n.4, p.25-49, 2014.

BELUCCI JUNIOR, J. A.; VITURI, D. W.; VERSA, G. L. G. S.; FURUYA, P. S.; VIDOR. R. C.; MATSUDA. L. M. Acolhimento com classificação de risco em serviço hospitalar de emergência: avaliação do processo de atendimento. Rev Enferm UERJ. v.23, n.1, p. 82-7, 2015.

BRASIL (BR). Ministério da Saúde. Conselho Nacional de Saúde. Resolução 196, de 10 de outubro de 1996: diretrizes e normas regulamentadoras de pesquisa envolvendo seres humanos. Brasília; 1996.

BRASIL (BR). Ministério da Saúde. Portaria N. ${ }^{02} 2048 /$ GM de 5 de novembro de 2002. Disponível em: http://dtr2001. saude.gov.br/sas/PORTARIAS/Port2002/Gm/GM-2048. htm. Acesso em 2009 Abr 11.

BRASIL (BR). Ministério da Saúde. Secretaria Executiva, Núcleo Técnico da Política Nacional de Humanização. Humaniza SUS: acolhimento com avaliação e classificação de risco: um paradigma ético-estético no fazer em saúde. Brasília (DF): MS; 2004.

BRASIL (BR). Ministério da Saúde. Secretaria de Atenção à Saúde, Política Nacional de Humanização da Atenção e Gestão do SUS. Acolhimento e Classificação de Risco nos Serviços de Urgência, Série B. Textos Básicos em Saúde. Brasília (DF); MS; 2009.

FEIJÓ, V. B. R.; CORDONI JUNIOR. L.; SOUZA, R. K. T.; DIAS, A. O. Análise da demanda atendida em uma unidade de urgência com classificação de risco. Saúde Debate. v.39, n.106, p.627-36, 2015.

JUNIOR, J. A. B.; MATSUDA, L. S. Implantação do Sistema Acolhimento com Classificação e Avaliação de 
Risco e Uso de Fluxograma Analisador. Texto; Contexto

Enferm. v.21, n.1, p.217-25, 2012.

MINAYO, M.C.S. O Desafio do Conhecimento: pesquisa qualitativa em saúde. 11. ed. São Paulo: Hucitex, 2008.

MOURA, L. B. A.; STHEPAN, I. T. K.; HOEPFNER, N. O.; CALADO, J. G. I. Decifrando redes de proteção em um território: relato de experiência no programa Pro- Saúde. Tempus, Actas de Saúde Colet. v.9, n.1, p.137-44, 2015.

SOUZA, C. C.; TOLEDO, A. D.; TADEU. L. F. R.; CHIANCA, T. C. M. Classificação de risco em prontosocorro: concordância entre um protocolo institucional brasileiro e Manchester. Revista Latino-americana de Enfermagem. v.19, n.1, p.08Telas, 2011.

WEYKAMP, J. M. PICKERSGILL C. S.; CECAGNO, D.; VIEIRA, F. P.; SIQUEIRA, H. C. H. Acolhimento com classificação de risco nos serviços de urgência e emergência: aplicabilidade na enfermagem. Rev RENE. v.16, n.3, p.32736, 2015. 